\title{
Introduction of Substituents on the 2-Oxo-piperazine Skeleton by [3+2] Cycloaddition and Subsequent Transformation*
}

\author{
Frank Wierschem and Karola Rück-Braun \\ Institut für Chemie, Technische Universität Berlin, Sekr. C 3, Straße des 17. Juni 135, \\ D-10623 Berlin \\ Reprint requests to Prof. Dr. K. Rück-Braun. Fax: (+49) 30-314-79651. \\ E-mail: krueck@chem.tu-berlin.de
}

Z. Naturforsch. 61b, 431 - 436 (2006); received January 17, 2006

The 3,4-substituted 2-oxo-piperazines $\mathbf{5 - 9}$ are obtained by [3+2] cycloaddition from nitrone $\mathbf{1}$ and a variety of alkenes. Subsequent functionalization of the bicyclic adducts involves reductive $\mathrm{N}-\mathrm{O}$ bond cleavage. A route towards libraries of immobilized 1,3-aminoalcohols with a 3,4-substituted 2-oxo-piperazine scaffold is briefly discussed for adducts derived from $N$-substituted maleic imides.

Key words: 2-Oxo-piperazine, Scaffold, [3+2] Cycloaddition, Nitrone, N-O Bond Cleavage

\section{Introduction}

In medicinal and agricultural chemistry heterocycles are among the target molecules for synthesizing libraries of compounds for finding lead structures. This is also the case for 2-oxo-piperazines and derivatives, which are privileged biologically active scaffolds and promising pharmaceutical target molecules [1]. From a medicinal standpoint the fields of applications are numerous, e.g. 2-oxo-piperazines were successfully investigated as farnesyl-protein transferase inhibitors, fXa inhibitors or non-peptide GPII antagonists [2]. Members of this class of compounds were investigated as nucleoside analogs [3]. They were found to display antiviral activity against a mammalian retrovirus [4]. Furthermore, 2-oxo-piperazines are promising scaffolds in the treatment of asthma and bronchitis [5].

Recently, we have reported [3+2] cycloaddition reactions of a polymer-bound 2-oxo-piperazine-derived nitrone with alkenes and alkynes furnishing libraries of 3,4-disubstituted and 3,4,5-trisubstituted 2-oxopiperazines $[1,6]$. The bicyclic (2-oxo-piperazine)based isoxazolidines and isoxazolines are well suited for additional functionalization steps starting with $\mathrm{N}-\mathrm{O}$ bond cleavage furnishing versatile libraries of 2-oxo-piperazines.

\footnotetext{
* Presented in part at the $7^{\text {th }}$ Conference on Iminium Salts
} (ImSaT-7), Bartholomä/Ostalbkreis, September 6-8, 2005.
In this paper we describe exemplarily details for the preparation of 3,4-disubstituted 2-oxo-piperazines in solution. In addition, we present key steps for the preparation of libraries of functionalized 1,3aminoalcohols from maleic imide-based cycloadducts by reductive $\mathrm{N}-\mathrm{O}$ bond cleavage on solid support using $\mathrm{Mo}(\mathrm{CO})_{6}$.

\section{Results and Discussion}

For the $[3+2]$ cycloadditions in solution described here the nitrone $\mathbf{1}$ was synthesized starting from 2oxo-piperazine 2 by alkylation of the amide with bromoacetic acid methyl ester followed by deprotection of the Boc-protecting group using trifluoroacetic acid in anisole. The secondary amine was generated prior to oxidation by adjusting a $\mathrm{pH}$-value of 7.5 using triethylamine. Subsequent treatment with $30 \%$ aqueous $\mathrm{H}_{2} \mathrm{O}_{2}$ (2.2 equiv.) using $\mathrm{Na}_{2} \mathrm{WO}_{4} \times 2 \mathrm{H}_{2} \mathrm{O}$ in methanol as catalyst gave nitrone $\mathbf{1}$ in $73 \%$ isolated yield (Scheme 1) [7]. This proved to be the method of choice, since oxidation with Davis' Reagent [7] (2.0 equiv.) in either $\mathrm{CH}_{2} \mathrm{Cl}_{2}$ or $\mathrm{CHCl}_{3}$ yielded nitrone $\mathbf{1}$ only in 32 and $34 \%$ yield, respectively.

Starting from $5 \mathrm{H}$-furan-2-one and crotonic acid methyl ester the expected cycloadducts 5 and $\mathbf{6}$ (Scheme 2) were isolated in thermal reactions in good yields, although long reaction times were required (5: 56 h, 6: 42 h). Notably, $\mathrm{Yb}(\mathrm{OTf})_{3} \times \mathrm{H}_{2} \mathrm{O}$-catalyzed reactions $(20 \mathrm{~mol} \%)$ could be successfully applied to 


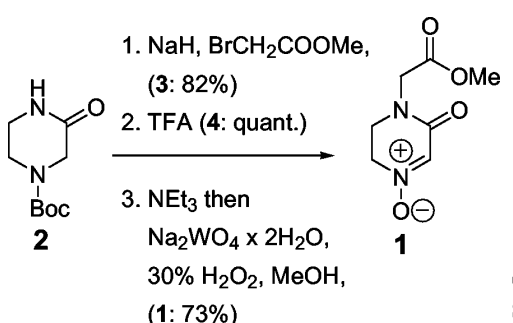

(1: $73 \%)$

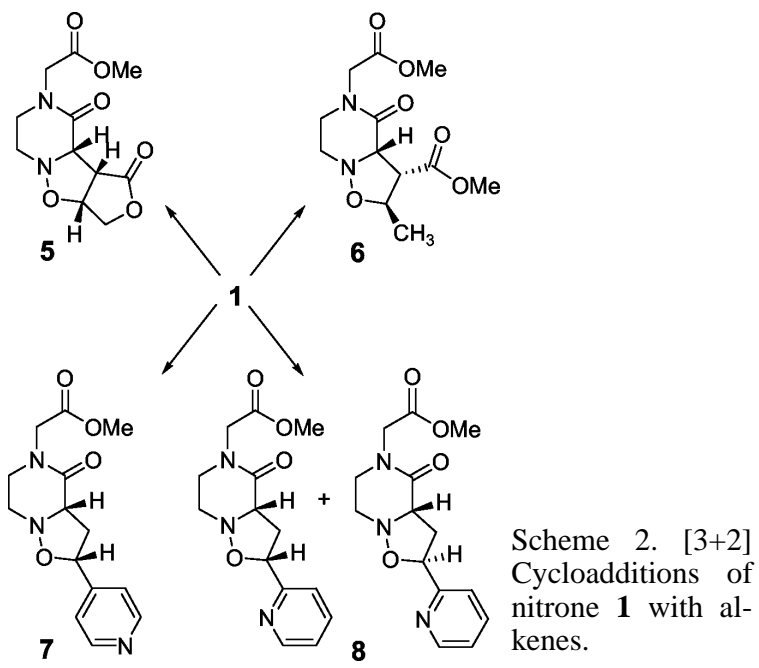

shorten the reaction times $(5: 4 \mathrm{~h}, \mathbf{6 :} 14 \mathrm{~h})$. The endoadducts were obtained regioselectively, as confirmed by NOE experiments.

The endo-preference is also very high for [3+2] cycloaddition reactions using 2-vinylpyridine and 4vinylpyridine (Scheme 2). Only a small amount of exo-8 was detected and structurally assigned. In test reactions we also examined $N$-phenylmaleic imide as dipolarophile (Scheme 3). The reaction proceeded regioselectively, the resulting adduct 9 was isolated in $66 \%$ yield and was treated with $\mathrm{Mo}(\mathrm{CO})_{6}$ for cleavage of the $\mathrm{N}-\mathrm{O}$ bond to obtain the 1,3-aminoalcohol 10 (Scheme 3).

Usually $\mathrm{Pd} / \mathrm{C}, \mathrm{Pd}(\mathrm{OH})_{2} / \mathrm{C}$ or Raney nickel in the presence of $\mathrm{H}_{2}$, or zinc in acetic acid are used for $\mathrm{N}-\mathrm{O}$ bond cleavage in solution phase studies [8]. However, for the preparation of libraries of compounds on solid support using polystyrene resins these methods are not suitable, because of the heterogeneous reaction conditions or insufficient swelling of the resins. For automated solid phase synthesis we also needed to avoid reaction conditions under pressure. Therefore, we turned our attention to a $\mathrm{Mo}(\mathrm{CO})_{6}$-mediated (0.7-6 equiv.) reductive cleavage of the $\mathrm{N}-\mathrm{O}$ bond in wet acetoni-

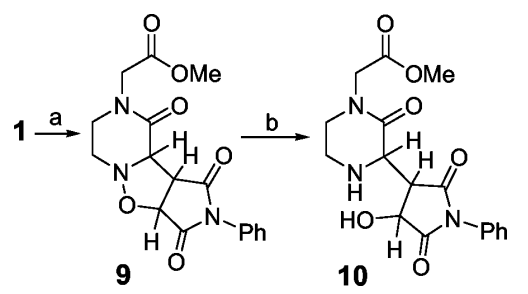

Scheme 3. Conditions: (a) $N$-Phenylmaleic imide ( 3 equiv.), $\mathrm{THF}, 65^{\circ} \mathrm{C}$, (b) $\mathrm{Mo}(\mathrm{CO})_{6}$ ( 3 equiv.), $\mathrm{MeCN} / \mathrm{H}_{2} \mathrm{O}, 85^{\circ} \mathrm{C}$.

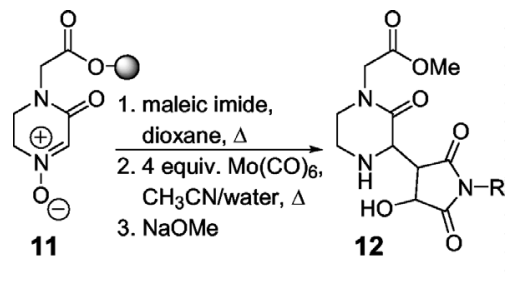

12a: $\mathrm{R}=3-\mathrm{Cl}, 5-\mathrm{Cl}-\mathrm{C}_{6} \mathrm{H}_{3}$

Scheme 4. Automated synthesis of 1,3-aminoalcohols 12: $\mathrm{R}=\mathrm{Me}(56 \%), \mathrm{Et}(62 \%), \operatorname{Pr}(75 \%), \mathrm{C}_{6} \mathrm{H}_{11}(81 \%), \mathrm{CH}_{2} \mathrm{C}_{6} \mathrm{H}_{5}$ $(70 \%), \mathrm{C}_{6} \mathrm{H}_{5}(65 \%), 12 \mathrm{a}: 3-\mathrm{Cl}, 5-\mathrm{Cl}-\mathrm{C}_{6} \mathrm{H}_{3}(90 \%)$.

trile at $85{ }^{\circ} \mathrm{C}$ [9]. The aminoalcohol $\mathbf{1 0}$ was obtained in $38 \%$ yield (Scheme 3 ). Unfortunately, the purification proceeded sluggishly. Generally, only after several manipulations, including separation of the impurities derived from $\mathrm{Mo}(\mathrm{CO})_{6}$ by hyflow ${ }^{\circledR}$ filtrations, precipitation with ether and a flash-column chromatography prior to a preparative RP-HPLC, pure product was isolated $[1,9]$. However, solid phase synthesis can result in an easier purification of intermediates, by removing the excess of reagents applied by simple washing procedures prior to cleavage from the solid. Consequently, we turned our attention next to automated solid phase synthesis without further optimization of solution phase studies.

The polymer-bound nitrone $\mathbf{1 1}$ was prepared as previously described [1]. The compound immobilized on Wang resin is stable at r.t. and can be stored on the bench for several months without decomposition. For the synthesis of a library we employed seven $\mathrm{N}$ substituted maleic imides. The reactions were carried out in a myriad core system (Mettler Toledo) in dioxane at $65{ }^{\circ} \mathrm{C}$. All cycloadditions proceeded smoothly, as well as the subsequent reductive $\mathrm{N}-\mathrm{O}$ bond cleavage with 4 equivalents of $\mathrm{Mo}(\mathrm{CO})_{6}$ followed by cleavage of the 1,3-aminoalcohols from Wang resin using $\mathrm{NaOMe}$.

The products were isolated in good to excellent yields (Scheme 4) compared with solution phase studies. During workup we observed the elimination of 
water by treatment with $\mathrm{NaOMe}$ in $\mathrm{MeOH} / \mathrm{THF}$ and subsequent evaporation of the solvent with a speed vac concentrator at $60{ }^{\circ} \mathrm{C}$. Thus, we obtained the corresponding unsaturated maleic imides as the only byproducts in minor amounts (analytical RP-HPLC/ MS). Byproduct formation can be avoided by evaporation of the solvent at lower temperature $\left(20{ }^{\circ} \mathrm{C}\right)$. Pure aminoalcohols were isolated after cleavage from the resin by a single purification step using preparative RPHPLC.

In conclusion, we have demonstrated a preparatively useful access to 3,4-disubstituted 2-oxo-piperazine templates and successfully proven the use of $\mathrm{Mo}(\mathrm{CO})_{6}$ for N-O bond cleavage in automated solid phase synthesis. This route has already been developed further beneficially as a starting point for more complex transformations for library generation $[1,6]$.

\section{Experimental Section}

\section{General information}

Solvents for the automated synthesis on solid support were used in p.a. quality without further purification. All products were characterized with RP-HPLC/LC-MS after cleavage from the resin. NMR spectra were measured on Bruker spectrometers AC200, AC400, AM400 and DRX500 at 200,400 and $500 \mathrm{MHz}$ for ${ }^{1} \mathrm{H}$ and 50.3, 100.6 and 125.7 $\mathrm{MHz}$ for ${ }^{13} \mathrm{C}$ at room temperature. Starting materials, reagents and compound $\mathbf{1 0}$ were prepared by following the literature procedure [1], and products were purified and analyzed by RP-HPLC as previously described by us.

1-Methoxycarbonylmethyl-4-tert-butyloxycarbonyl-2oxo-piperazine (3): Sodium hydride $(1.73 \mathrm{~g}, 73.0 \mathrm{mmol}$, 1.15 equiv.) was added in one portion at $0{ }^{\circ} \mathrm{C}$ to a stirred solution of 2 (12.70 g, $63.0 \mathrm{mmol}, 1.0$ equiv.) in $280 \mathrm{ml}$ of DMF. The reaction mixture was maintained at this temperature for $1 \mathrm{~h}$ until bromoacetic acid methylester $(6.60 \mathrm{ml}$, $69.0 \mathrm{mmol}, 1.10$ equiv.) was added. After $20 \mathrm{~min}$ at $0{ }^{\circ} \mathrm{C}$ the solution was allowed to warm to r.t. and stirring was continued (18 h, TLC monitoring). Then methanol (10 ml), water $(10 \mathrm{ml})$ and brine $(180 \mathrm{ml})$ were added. The organic layer was separated and the aqueous layer was extracted with diethyl ether $(1 \times 250 \mathrm{ml}, 3 \times 140 \mathrm{ml})$. The combined organic phase was dried $\left(\mathrm{MgSO}_{4}\right)$, filtered and concentrated in vacuo. Purification by flash chromatography on silica (pentane/acetone $4: 1 \rightarrow 2: 1)$ afforded $3(14.16 \mathrm{~g}, 82 \%$, $52.0 \mathrm{mmol})$ as a white solid. - M. p. $57^{\circ} \mathrm{C} .-R_{f}=0.60$ (pentane/acetone 1:1). $-{ }^{1} \mathrm{H}$ NMR (400 $\left.\mathrm{MHz}, \mathrm{CDCl}_{3}\right)$ : $\delta=4.15\left(\mathrm{~s}, 2 \mathrm{H} ; \mathrm{NCH}_{2} \mathrm{COOCH}_{3}\right), 4.11\left(\mathrm{~s}, 2 \mathrm{H} ; \mathrm{O}=\mathrm{C}-\mathrm{CH}_{2}\right.$ $\mathrm{N}), 3.72\left(\mathrm{~s}, 3 \mathrm{H} ; \mathrm{CH}_{3}\right), 3.69-3.66\left(\mathrm{t}, 2 \mathrm{H},{ }^{3} \mathrm{~J}=5.5 \mathrm{~Hz} ; \mathrm{CH}_{2}\right)$, $3.42-3.39$ (t, $\left.2 \mathrm{H},{ }^{3} \mathrm{~J}=5.5 \mathrm{~Hz} ; \mathrm{CH}_{2}\right), 1.44$ (s, 9H; $\left.\mathrm{CH}_{3}\right)$. ${ }^{13} \mathrm{C}$ NMR (50.3 MHz, $\left.\mathrm{CDCl}_{3}\right): \delta=169.0(\mathrm{C}=\mathrm{O}), 166.3$
$(\mathrm{C}=\mathrm{O}), 153.7(\mathrm{C}=\mathrm{O}), 80.8\left(\mathrm{C}_{\left.\left(\mathrm{CH}_{3}\right)_{3}\right),}, \mathrm{C2.2}\left(\mathrm{C}=\mathrm{O}-\mathrm{OCH}_{3}\right)\right.$, $48.0\left(\mathrm{CH}_{2}\right), 47.5\left(\mathrm{CH}_{2}\right), 40.0\left(\mathrm{br}, \mathrm{CH}_{2}\right), 28.2\left(\mathrm{C}\left(\mathrm{CH}_{3}\right)_{3}\right)$. IR (KBr): $v=2988,2954,2874,1758(\mathrm{C}=\mathrm{O}$, ester $), 1692$ (C=O, Boc), 1654 (C=O, 2-oxo-piperazine), 1493, 1480, 1422, 1367, 1337, 1322, 1293, 1239, 1133, 1099, 1073, 1011, 990, 979, 965, 868, $771 \mathrm{~cm}^{-1}$. - MS (EI, $\left.90^{\circ} \mathrm{C}\right): \mathrm{m} / \mathrm{z}$ $(\%)=272(1)\left[\mathrm{M}^{+}\right], 216(12), 172$ (20), 157 (8), 113 (16), 85 (8), 57 (100). - HR-MS (EI): calcd. 272.1372; found 272.1377. $-\mathrm{C}_{12} \mathrm{H}_{20} \mathrm{~N}_{2} \mathrm{O}_{5}$ (272.3): calcd. C 52.93, H 7.40, $\mathrm{N}$ 10.29; found C 52.90, H 7.34, N 10.15.

Compound 4: Trifluoroacetic acid $(48.2 \mathrm{ml}, 0.626 \mathrm{~mol}$, 16.50 equiv.) was added to a stirred solution of 3 (10.42 g, $38 \mathrm{mmol}, 1.0$ equiv.) in anisole $(86 \mathrm{ml})$. After $90 \mathrm{~min}$ (TLC monitoring) toluene $(85 \mathrm{ml})$ was added and the solvent was removed in vacuo. The crude product 4 was used in the next step without further purification (14.77 g, quant.), pale yellow solid. - M. p. $112{ }^{\circ} \mathrm{C} .-R_{f}=0.01$ (methanol). $-{ }^{1} \mathrm{H}$ NMR $\left(200 \mathrm{MHz}, \mathrm{CD}_{3} \mathrm{CN}\right): \delta=4.12$ (s, $2 \mathrm{H} ; \mathrm{NCH}_{2} \mathrm{COOCH}_{3}$ ), 3.81 (s, $\left.2 \mathrm{H} ; \mathrm{O}=\mathrm{C}-\mathrm{CH}_{2}-\mathrm{N}\right), 3.68\left(\mathrm{~s}, 3 \mathrm{H} ; \mathrm{CH}_{3}\right), 3.63-3.58(\mathrm{~m}, 2 \mathrm{H}$; $\mathrm{CH}_{2}$ ), $3.48-3.43$ (m, 2H; $\left.\mathrm{CH}_{2}\right) .-{ }^{13} \mathrm{C}$ NMR (50.3 MHz, $\left.\mathrm{CD}_{3} \mathrm{CN}\right): \delta=169.9(\mathrm{C}=\mathrm{O}), 163.1(\mathrm{C}=\mathrm{O}), 161.3\left(\mathrm{q},{ }^{2} \mathrm{~J}=\right.$ $\left.36.1 \mathrm{~Hz} ; \mathrm{F}_{3} \mathrm{CCOO}^{-}\right), 52.9\left(\mathrm{C}=\mathrm{O}-\mathrm{OCH}_{3}\right), 48.7,45.6,45.1$, $41.6\left(\mathrm{CH}_{2}\right)$. The signals of the $\mathrm{C}$-atom of the $\mathrm{CF}_{3}$-group are not detectable. - IR (KBr): $v=3017,2965,2828,2767$, 2676, 2594, 2491, 1752 (C=O, ester), $1672\left(\mathrm{~F}_{3} \mathrm{CC}=\mathrm{O}\right), 1659$ (C=O, 2-oxo-piperazine), 1504, 1483, 1472, 1428, 1402, 1354, 1296, 1261, 1230, 1203, 1193, 1169, 1143, 1080, 1021, 837, 799, 723, 685, $515 \mathrm{~cm}^{-1}$. - MS (EI, $\left.120^{\circ} \mathrm{C}\right): \mathrm{m} / \mathrm{z}$ $(\%)=172(92)\left[\mathrm{M}^{+}-\mathrm{F}_{3} \mathrm{CCOOH}\right], 113(12)\left[\mathrm{F}_{3} \mathrm{CCOO}^{-}\right]$, 102 (40), 85 (100), 69 (40), 56 (88), 57 (32), 51 (24). - HRMS (EI): calcd. for $\mathrm{C}_{7} \mathrm{H}_{12} \mathrm{~N}_{2} \mathrm{O}_{3}$ 172.0847; found 172.0844.

Nitrone 1: Triethylamine $(5.37 \mathrm{ml}, 14.20 \mathrm{mmol}, 1.1$ equiv.) was added dropwise to a suspension of $4(5.0 \mathrm{~g}$, $12.95 \mathrm{mmol}, 1.0$ equiv.) in methanol $(50 \mathrm{ml})$, until $\mathrm{pH} 7.5$ was adjusted. Then the reaction mixture was stirred at r.t. $(1 \mathrm{~h})$ before $\mathrm{Na}_{2} \mathrm{WO}_{4} \times 2 \mathrm{H}_{2} \mathrm{O}(0.29 \mathrm{~g}, 0.65 \mathrm{mmol}, 0.05$ equiv.) was added in one portion. After cooling the reaction mixture to $0{ }^{\circ} \mathrm{C}, 30 \%$ aqueous $\mathrm{H}_{2} \mathrm{O}_{2}(3.92 \mathrm{ml}, 28.50 \mathrm{mmol}$, 2.2 equiv.) was added $(10 \mathrm{~min})$. The reaction mixture was stirred at $0{ }^{\circ} \mathrm{C}(15 \mathrm{~min})$ and after warming to r.t. stirring was continued until TLC monitoring indicated complete consumption of the starting material ( $2 \mathrm{~h} 45 \mathrm{~min})$. The solvent was removed in vacuo and the remaining residue was dissolved in dichloromethane $(40 \mathrm{ml})$. The organic layer was washed with brine $(2 \times 40 \mathrm{ml})$ and then the aqueous layer extracted with dichloromethane. The combined organic layer was dried $\left(\mathrm{MgSO}_{4}\right)$ and the solvent removed in vacuo yielding $5.78 \mathrm{~g}$ of $\mathbf{1}$ as a pale yellow oil. Purification by flash chromatography on silica (ethyl acetate/methanol $80: 1$ ) afforded $1(1.76 \mathrm{~g}, 73 \%, 9.45 \mathrm{mmol})$ as a white solid. - M. p. $78^{\circ} \mathrm{C}$. $R_{f}=0.68$ (methanol). $-{ }^{1} \mathrm{H} \mathrm{NMR}\left(200 \mathrm{MHz}, \mathrm{CDCl}_{3}\right): \delta=$ $7.17(\mathrm{~s}, 1 \mathrm{H} ; \mathrm{N}=\mathrm{CH}), 4.23\left(\mathrm{~s}, 2 \mathrm{H} ; \mathrm{CH}_{2}\right), 4.19-4.13(\mathrm{t}, 2 \mathrm{H}$, $\left.{ }^{3} J=6.35 \mathrm{~Hz} ; \mathrm{CH}_{2}\right), 3.78-3.72\left(\mathrm{t}, 2 \mathrm{H},{ }^{3} J=6.35 \mathrm{~Hz} ; \mathrm{CH}_{2}\right)$, 
3.75 (s, 3H; $\left.\mathrm{CH}_{3}\right) .-{ }^{13} \mathrm{C}$ NMR (50.3 $\left.\mathrm{MHz}, \mathrm{CDCl}_{3}\right): \delta=$ $168.6(\mathrm{C}=\mathrm{O}), 159.3(\mathrm{C}=\mathrm{O}), 128.4(\mathrm{C}=\mathrm{N}), 58.7\left(\mathrm{CH}_{2}\right), 52.3$ $\left(\mathrm{CH}_{3}\right), 46.9,43.8\left(\mathrm{CH}_{2}\right) .-\mathrm{IR}(\mathrm{KBr}): v=3059,2991,1758$ $(\mathrm{C}=\mathrm{O}$, ester), $1652(\mathrm{C}=\mathrm{O}, 2$-oxo-piperazine $), 1574(\mathrm{C}=\mathrm{N})$, $1488,1457,1432,1399,1365,1349,1339,1305,1282$, $1250,1214,1171,1131,1085,1023,986,970,932,884$, $845,722,694 \mathrm{~cm}^{-1}$. - MS (EI, $\left.60{ }^{\circ} \mathrm{C}\right): \mathrm{m} / \mathrm{z}(\%)=186(78)$ $\left[\mathrm{M}^{+}\right], 127(100)\left[\mathrm{M}^{+}-\mathrm{CO}_{2} \mathrm{CH}_{3}\right], 116(14), 69$ (86), 56 (40), 51 (42). - HR-MS (EI): calcd. 186.0640; found 186.0639. $\mathrm{C}_{7} \mathrm{H}_{10} \mathrm{~N}_{2} \mathrm{O}_{4}$ (186.17): calcd. C 45.16, H 5.41, N 15.04; found $\mathrm{C} 45.09$, H 5.48, N 15.17 .

\section{[3+2] Cycloaddition reactions of nitrone $\mathbf{1}$ with alkenes in} solution

General procedure: Nitrone 1 was dissolved in THF and the alkene (3.0 equiv.) was added. Then the reaction mixture was heated to reflux until TLC monitoring indicated complete consumption of the starting material. The solvent was removed in vacuo and the resulting crude product was purified by flash chromatography on silica or by preparative RPHPLC.

Cycloadduct (5): yellow solid. - M.p. $160{ }^{\circ} \mathrm{C} .-{ }^{1} \mathrm{H}$ NMR $\left(200 \mathrm{MHz}, \mathrm{CDCl}_{3}\right): \delta=4.97-4.89$ (dddd, $1 \mathrm{H},{ }^{3} \mathrm{~J}=$ $\left.7.28 \mathrm{~Hz},{ }^{3} J=5.5 \mathrm{~Hz},{ }^{3} J=1.9 \mathrm{~Hz},{ }^{4} J=1.2 \mathrm{~Hz} ; 8-\mathrm{H}\right), 4.53-$ $4.39\left(\mathrm{~m}, 2 \mathrm{H},{ }^{3} J=5.5 \mathrm{~Hz},{ }^{3} J=1.9 \mathrm{~Hz}\right.$; 9-H, 9'-H), 4.29 $\left(\mathrm{d}, 1 \mathrm{H},{ }^{2} J=17.08 \mathrm{~Hz} ; \mathrm{NCH}_{2} \mathrm{COOCH}_{3}\right), 4.25\left(\mathrm{~d}, 1 \mathrm{H},{ }^{3} \mathrm{~J}=\right.$ $2.44 \mathrm{~Hz}$; 3-H), $4.07-3.93(\mathrm{~m}, 2 \mathrm{H} ; 6-\mathrm{H}, 7-\mathrm{H}), 3.96(\mathrm{~d}, 1 \mathrm{H}$, $\left.{ }^{2} J=17.08 \mathrm{~Hz} ; \mathrm{NCH}_{2} \mathrm{COOCH}_{3}\right), 3.73\left(\mathrm{~s}, 3 \mathrm{H} ; \mathrm{CH}_{3}\right), 3.52-$ $3.43(\mathrm{~m}, 2 \mathrm{H} ; 5-\mathrm{H}, 5$ '-H), 3.16-3.07 (ddd, $1 \mathrm{H}, J=1.96$, 4.40, $5.36 \mathrm{~Hz} ; 6$ '-H). $-{ }^{13} \mathrm{C} \mathrm{NMR}\left(50.3 \mathrm{MHz}, \mathrm{CDCl}_{3}\right): \delta=$ $175.5(\mathrm{C}=\mathrm{O} ; \mathrm{C}-11), 168.5\left(\mathrm{C}=\mathrm{O} ; \mathrm{NCH}_{2} \mathrm{COOCH}_{3}\right), 166.7$ $(\mathrm{C}=\mathrm{O} ; \mathrm{C}-2), 75.4(\mathrm{C}-8), 74.2(\mathrm{C}-9), 66.6(\mathrm{C}-3), 52.2\left(\mathrm{CH}_{3}\right)$, $51.6(\mathrm{C}-7), 48.5\left(\mathrm{NCH}_{2} \mathrm{COOCH}_{3}\right), 46.2$ (C-5), 43.3 (C-6). For the signal assignments ${ }^{1} \mathrm{H},{ }^{1} \mathrm{H}-\mathrm{COSY}, \mathrm{HMBC}, \mathrm{NOE}$ and HMQC spectra were recorded. - IR (KBr): $v=2982,2969$, 2949, $1774(\mathrm{C}=\mathrm{O}, \mathrm{C}-11), 1742(\mathrm{C}=\mathrm{O}$, ester $), 1652(\mathrm{C}=\mathrm{O}$, 2-oxo-piperazine), 1502, 1461, 1399, 1389, 1380, 1359, $1338,1295,1219,1207,1189,1149,1075,1054,987,944$, 934, 902, 836, 731, 724, 575, 530, $491 \mathrm{~cm}^{-1}$. - MS (EI, $\left.120{ }^{\circ} \mathrm{C}\right): \mathrm{m} / \mathrm{z}(\%)=270(60)\left[\mathrm{M}^{+}+1\right], 211(100)\left[\mathrm{M}^{+}\right.$$\mathrm{COOCH}_{3}$ ], 183 (24), 158 (44), 125 (20), 123 (16), 56 (66), 55 (26). - HR-MS (EI): calcd. 270.0851; found 270.0853. $\mathrm{C}_{11} \mathrm{H}_{14} \mathrm{~N}_{2} \mathrm{O}_{6}$ (270.24): calcd. C 48.89, H 5.22, N 10.36; found C 48.70, H 5.43, N 9.92 .

Cycloadduct 6: yellow oil, 98\%. - ${ }^{1} \mathrm{H}$ NMR $(500 \mathrm{MHz}$, $\left.\mathrm{CDCl}_{3}\right): \delta=4.54-4.52(\mathrm{~m}, 1 \mathrm{H} ; 8-\mathrm{H}), 4.39(\mathrm{~d}, 1 \mathrm{H}$, $\left.{ }^{3} J=10.05 \mathrm{~Hz} ; 3-\mathrm{H}\right), 4.22\left(\mathrm{~d}, 1 \mathrm{H},{ }^{2} J=17.30 \mathrm{~Hz}\right.$; $\left.\mathrm{NCH}_{2} \mathrm{COOCH}_{3}\right), 3.97\left(\mathrm{~d}, 1 \mathrm{H},{ }^{2} J=17.30 \mathrm{~Hz} ; \mathrm{NCH}_{2}\right.$ $\left.\mathrm{COOCH}_{3}\right) 3.69\left(\mathrm{~s}, 3 \mathrm{H} ; \mathrm{COOCH}_{3}\right), 3.65\left(\mathrm{~s}, 3 \mathrm{H} ; \mathrm{COOCH}_{3}\right)$, $3.63-3.58(\mathrm{~m}, 1 \mathrm{H} ; 6-\mathrm{H}), 3.56-3.53(\mathrm{~m}, 1 \mathrm{H} ; 5-\mathrm{H}), 3.38-$ $3.35\left(\mathrm{~m}, 1 \mathrm{H} ; 5^{\prime}-\mathrm{H}\right), 3.32\left(\mathrm{~d}, 1 \mathrm{H},{ }^{3} J=10.05 \mathrm{~Hz} ; 7-\mathrm{H}\right), 3.21-$ $3.18\left(\mathrm{~m}, 1 \mathrm{H} ; 6{ }^{\prime}-\mathrm{H}\right), 1.34\left(\mathrm{~d}, 3 \mathrm{H},{ }^{3} J=6.00 \mathrm{~Hz} ; 9-\mathrm{H}\right)$. ${ }^{13} \mathrm{C}$ NMR $\left(50.3 \mathrm{MHz}, \mathrm{CDCl}_{3}\right): \delta=171.5(\mathrm{C}=\mathrm{O}), 168.5$
$(\mathrm{C}=\mathrm{O}), 166.2(\mathrm{C}=\mathrm{O}), 76.7(\mathrm{C}-8), 67.6(\mathrm{C}-3), 58.5(\mathrm{C}-7)$, $52.2\left(\mathrm{COOCH}_{3}\right), 52.0\left(\mathrm{COOCH}_{3}\right), 47.6\left(\mathrm{NCH}_{2} \mathrm{COOCH}_{3}\right)$, 47.1 (C-6), 44.9 (C-5), 18.4 (C-9). For the signal assignments ${ }^{1} \mathrm{H},{ }^{1} \mathrm{H}-\mathrm{COSY}$ and HMQC spectra were recorded. IR (ATR): $v=2955,1735(\mathrm{C}=\mathrm{O}), 1655(\mathrm{C}=\mathrm{O}), 1640(\mathrm{C}=\mathrm{O})$, $1491,1436,1404,1373,1344,1292,1277,1201,1179$ 1103, 1078, 1037, 998, 974, $938 \mathrm{~cm}^{-1}$. - MS (EI, $120^{\circ} \mathrm{C}$ ): $\mathrm{m} / \mathrm{z}(\%)=286(24)\left[\mathrm{M}^{+}\right], 255(20)\left[\mathrm{M}^{+}-\mathrm{OCH}_{3}\right], 211(58)$, 199 (26), 183 (50), 158 (100), 127 (16), 123 (30), 69 (30), 56 (66). - HR-MS (EI): calcd. for $\mathrm{C}_{12} \mathrm{H}_{18} \mathrm{~N}_{2} \mathrm{O}_{6}$ 286.1164; found 286.1165 .

Cycloadduct 7: By following the general procedure, nitrone $1(200 \mathrm{mg}, 1.07 \mathrm{mmol})$ dissolved in THF $(5.0 \mathrm{ml})$ was treated with 4-vinylpyridine $(0.35 \mathrm{ml}, 3.22 \mathrm{mmol}, 3.0$ equiv.) for $22 \mathrm{~h}$. Purification by flash column chromatography (pentane/acetone 4:1) afforded endo-7 [ $[3 S, 8 R)$ and $(3 R, 8 S)],(233 \mathrm{mg}, 75 \%, 0.80 \mathrm{mmol})$ as a yellow oil. $-R_{f}=$ 0.24 (acetone). $-{ }^{1} \mathrm{H}$ NMR $\left(200 \mathrm{MHz}, \mathrm{CDCl}_{3}\right): \delta=8.79$ $8.76\left(\mathrm{dd}, 2 \mathrm{H},{ }^{3} \mathrm{~J}=6.82 \mathrm{~Hz},{ }^{4} \mathrm{~J}=1.54 \mathrm{~Hz}\right.$; ortho-H), 7.79 (d, $2 \mathrm{H},{ }^{3} \mathrm{~J}=6.82 \mathrm{~Hz}$; meta-H), $5.41-5.33\left(\mathrm{dd}, 1 \mathrm{H},{ }^{3} \mathrm{~J}=\right.$ $\left.5.86 \mathrm{~Hz},{ }^{3} J=4.40 \mathrm{~Hz} ; 8-\mathrm{H}\right), 4.36\left(\mathrm{~d}, 1 \mathrm{H},{ }^{2} J=17.56 \mathrm{~Hz}\right.$; $\left.\mathrm{NCH}_{2} \mathrm{COOCH}_{3}\right), 4.16-4.10\left(\mathrm{dd}, 1 \mathrm{H},{ }^{3} J=2.92 \mathrm{~Hz},{ }^{3} \mathrm{~J}=\right.$ $8.30 \mathrm{~Hz} ; 3-\mathrm{H}), 4.05-3.93(\mathrm{~m}, 1 \mathrm{H} ; 5-\mathrm{H}), 4.04-3.95(\mathrm{~d}$, $\left.1 \mathrm{H},{ }^{2} \mathrm{~J}=17.56 \mathrm{~Hz} ; \mathrm{NCH}_{2} \mathrm{COOCH}_{3}\right), 3.76\left(\mathrm{~s}, 3 \mathrm{H} ; \mathrm{CH}_{3}\right)$, $3.67-3.46\left(\mathrm{~m}, 2 \mathrm{H} ; 6-\mathrm{H}, 6{ }^{\prime}-\mathrm{H}\right), 3.35-3.21(\mathrm{~m}, 2 \mathrm{H} ; 7-\mathrm{H}$, $\left.5^{\prime}-\mathrm{H}\right), 2.66-2.52\left(\mathrm{ddd}, 1 \mathrm{H},{ }^{3} J=5.86 \mathrm{~Hz},{ }^{3} J=8.30 \mathrm{~Hz}\right.$, $\left.{ }^{2} J=14.10 \mathrm{~Hz} ; 7^{\prime}-\mathrm{H}\right) .-{ }^{13} \mathrm{C}$ NMR $\left(125 \mathrm{MHz}, \mathrm{CDCl}_{3}\right)$ : $\delta=168.9\left(\mathrm{C}=\mathrm{O} ; \mathrm{NCH}_{2} \mathrm{COOCH}_{3}\right), 168.6(\mathrm{C}=\mathrm{O} ; \mathrm{C}-2), 160.5$ (C-quart), 143.3 (ortho-C), 123.0 (meta-C), 75.8 (C-8), 63.1 $(\mathrm{C}-3), 52.4\left(\mathrm{COOCH}_{3}\right), 48.5\left(\mathrm{NCH}_{2} \mathrm{COOCH}_{3}\right), 47.4(\mathrm{C}-6)$, $43.6(\mathrm{C}-5), 41.8(\mathrm{C}-7)$. For the signal assignments ${ }^{1} \mathrm{H},{ }^{1} \mathrm{H}-$ COSY, HMBC, NOE and HMQC spectra were recorded. IR (ATR): $v=2954,2855,1748(\mathrm{C}=\mathrm{O}$, ester $), 1655(\mathrm{C}=\mathrm{O}, 2$ oxo-piperazine), 1600, 1560, 1491, 1438, 1412, 1364, 1345, 1290, 1214, 1182, 1067, 994, $815 \mathrm{~cm}^{-1}$. - MS (EI, $\left.120{ }^{\circ} \mathrm{C}\right)$ : $\mathrm{m} / \mathrm{z}(\%)=292(12)\left[\mathrm{M}^{+}+1\right], 291(100)\left[\mathrm{M}^{+}\right], 274(44), 204$ (28), 158 (68), 125 (76), 106 (40), 69 (44), 56 (92). - HRMS(EI): calcd. 291.1219; found 291.1221. $-\mathrm{C}_{14} \mathrm{H}_{17} \mathrm{~N}_{3} \mathrm{O}_{4}$ (291.31): calcd. C 57.72, H 5.88, N 14.42; found C 57.81, H 6.01, N 14.25 .

Cycloadduct 8: By following the general procedure, nitrone $1(250 \mathrm{mg}, 1.34 \mathrm{mmol})$ dissolved in THF $(5.0 \mathrm{ml})$ was treated with 2-vinylpyridine $(0.43 \mathrm{ml}, 4.03 \mathrm{mmol}, 3.0$ equiv.) for $23 \mathrm{~h}$. Purification by flash column chromatography (pentane/acetone $4: 1 \rightarrow$ acetone) afforded endo-8 $[(3 S, 8 R)$ and $(3 R, 8 S)]$, and exo-8 $[(3 R, 8 R)$ and $(3 S, 8 S)]$ : endo-8 (285 mg, $73 \%, 0.98 \mathrm{mmol})$, yellow oil. $-R_{f}=$ 0.57 (acetone). $-{ }^{1} \mathrm{H}$ NMR (200 MHz, $\left.\mathrm{CDCl}_{3}\right): \delta=8.53$ (d, $1 \mathrm{H},{ }^{3} J=4.88 \mathrm{~Hz}$; ortho-H), $7.73-7.64(\mathrm{~m}, 1 \mathrm{H}$; para$\mathrm{H}), 7.48\left(\mathrm{~d}, 1 \mathrm{H},{ }^{3} \mathrm{~J}=7.80 \mathrm{~Hz}\right.$; meta $\left.-\mathrm{H}\right), 7.23-7.16(\mathrm{~m}$, $1 \mathrm{H} ;$ meta-H), $5.21-5.12\left(\mathrm{~m}, 1 \mathrm{H},{ }^{3} J=8.30 \mathrm{~Hz} ; 8-\mathrm{H}\right), 4.28$ $\left(\mathrm{d}, 1 \mathrm{H},{ }^{2} \mathrm{~J}=17.56 \mathrm{~Hz} ; \mathrm{NCH}_{2} \mathrm{COOCH}_{3}\right), 4.26-4.18$ (dd, $\left.1 \mathrm{H},{ }^{3} J=6.34 \mathrm{~Hz},{ }^{3} \mathrm{~J}=6.80 \mathrm{~Hz} ; 3-\mathrm{H}\right), 4.01\left(\mathrm{~d}, 1 \mathrm{H},{ }^{2} J=\right.$ 
$\left.17.56 \mathrm{~Hz} ; \mathrm{NCH}_{2} \mathrm{COOCH}_{3}\right), 3.90-3.78(\mathrm{~m}, 1 \mathrm{H} ; 5-\mathrm{H}), 3.71$ (s, 3H; $\left.\mathrm{COOCH}_{3}\right), 3.60-3.39$ (m, 2H; 6-H, 6'-H), 3.353.09 (m, 2H; 7-H, 5'-H), 2.76-2.62 (ddd, $1 \mathrm{H}^{3} \mathrm{~J}=6.80 \mathrm{~Hz}$, $\left.{ }^{3} J=8.30 \mathrm{~Hz},{ }^{2} J=13.3 \mathrm{~Hz} ; 7^{\prime}-\mathrm{H}\right) .-{ }^{13} \mathrm{C}$ NMR $(125 \mathrm{MHz}$, $\left.\mathrm{CDCl}_{3}\right): \delta=169.3\left(\mathrm{C}=\mathrm{O}, \mathrm{NCH}_{2} \mathrm{COOCH}_{3}\right), 169.0(\mathrm{C}=\mathrm{O}$, 2-oxo-piperazine), 159.1 (q; C-aromat), 149.1 (ortho-C), 137.0 (para-C), 122.9 (meta-C), 121.2 (meta-C), 80.6 (C-8), $63.9(\mathrm{C}-3), 52.3\left(\mathrm{COOCH}_{3}\right), 48.6\left(\mathrm{NCH}_{2} \mathrm{COOCH}_{3}\right), 48.2$ (C-6), 44.2 (C-5), 40.4 (C-7). - IR (ATR): $v=2955,1746$ ( $\mathrm{C}=\mathrm{O}$, ester), 1646 ( $\mathrm{C}=\mathrm{O}, 2$ 2-oxo-piperazine), 1592, 1489, $1437,1405,1345,1210,1182,994,947,779,750 \mathrm{~cm}^{-1}$. MS (EI, $\left.130{ }^{\circ} \mathrm{C}\right): m / z(\%)=291(18)\left[\mathrm{M}^{+}\right], 184(28)$, 170 (20), 125 (24), 122 (56), 108 (100), 97 (28), 69 (24), 56 (56). - HR-MS (EI): calcd. 291.1219; found 291.1221. $\mathrm{C}_{14} \mathrm{H}_{17} \mathrm{~N}_{3} \mathrm{O}_{4}$ (291.31): calcd. C 57.72, H 5.88, N 14.42; found $\mathrm{C} 57.93, \mathrm{H} 6.07, \mathrm{~N} 14.09$; exo-8 $(54 \mathrm{mg}, 14 \%$, $0.19 \mathrm{mmol}$ ), yellow oil. $-R_{f}=0.27$ (acetone). $-{ }^{1} \mathrm{H}$ NMR

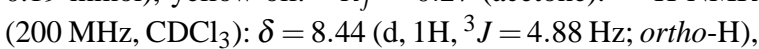
$7.63-7.54\left(\mathrm{dd}, 1 \mathrm{H},{ }^{3} J=7.80 \mathrm{~Hz},{ }^{3} J=7.82 \mathrm{~Hz}\right.$; para-H), $7.39\left(\mathrm{~d}, 1 \mathrm{H},{ }^{3} \mathrm{~J}=7.82 \mathrm{~Hz}\right.$; meta-H), $7.12-7.05(\mathrm{~m}, 1 \mathrm{H}$; meta-H), $5.27-5.20\left(\mathrm{~m}, 1 \mathrm{H},{ }^{3} J=4.88 \mathrm{~Hz} ; 8-\mathrm{H}\right), 4.23$ $\left(\mathrm{d}, 1 \mathrm{H},{ }^{2} J=17.56 \mathrm{~Hz} ; \mathrm{NCH}_{2} \mathrm{COOCH}_{3}\right), 4.21-4.14(\mathrm{~m}$, $1 \mathrm{H} ; 3-\mathrm{H}), 4.13\left(\mathrm{~d}, 1 \mathrm{H},{ }^{2} \mathrm{~J}=17.56 \mathrm{~Hz} ; \mathrm{NCH}_{2} \mathrm{COOCH}_{3}\right)$, $3.83-3.72(\mathrm{~m}, 1 \mathrm{H} ; 5-\mathrm{H}), 3.75\left(\mathrm{~s}, 3 \mathrm{H} ; \mathrm{COOCH}_{3}\right), 3.52-$ 3.33 (m, 3H; 5'-H, 6-H, 6'-H), $3.21-3.07$ (m, 1H; 7-H), $2.98-2.85\left(\mathrm{~m}, 1 \mathrm{H} ; 7^{\prime}-\mathrm{H}\right) .-{ }^{13} \mathrm{C} \mathrm{NMR}\left(125 \mathrm{MHz}, \mathrm{CDCl}_{3}\right)$ : $\delta=169.0\left(\mathrm{C}=\mathrm{O}, \mathrm{NCH}_{2} \mathrm{COOCH}_{3}\right), 168.8(\mathrm{C}=\mathrm{O}, 2$-oxopiperazine), 160.3 (q; C-aromat), 147.7 (ortho-C), 138.5 (para-C), 123.1 (meta-C), 121.1 (meta-C), 77.8 (C-8), 63.4 (C-3), $52.4\left(\mathrm{COOCH}_{3}\right), 48.2\left(\mathrm{NCH}_{2} \mathrm{COOCH}_{3}\right), 48.1$ (C-6), 44.2 (C-5), 40.5 (C-7). - IR (ATR): $v=2954,1747$ (C=O, ester), 1654 (C=O, 2-oxo-piperazine), 1591, 1570, 1491, 1473, 1437, 1404, 1364, 1344, 1291, 1213, 1182, 1152, 1046, 994, 835, 780, $751 \mathrm{~cm}^{-1}$. - MS (EI, $\left.140{ }^{\circ} \mathrm{C}\right): \mathrm{m} / \mathrm{z}$ $(\%)=292(8)\left[\mathrm{M}^{+}+1\right], 291(24)\left[\mathrm{M}^{+}\right], 274(12), 273(20)$, 260 (12), 185 (92), 184 (56), 125 (30), 117 (32), 108 (100), 56 (26). - HR-MS (EI): calcd. for $\mathrm{C}_{14} \mathrm{H}_{17} \mathrm{~N}_{3} \mathrm{O}_{4}$ : 291.1219; found 291.1223 .

Cycloadduct 9: By following the general procedure, nitrone $1(250 \mathrm{mg}, 1.34 \mathrm{mmol})$ dissolved in THF $(4.0 \mathrm{ml})$ was treated with $N$-phenylmaleic imide $(0.70 \mathrm{~g}, 4.02 \mathrm{mmol}$, 3.0 equiv.) dissolved in THF ( $1 \mathrm{ml})$ for $2 \mathrm{~h}$. Purification by flash chromatography (pentane/ethyl acetate $2: 1 \rightarrow 1: 1$ ) afforded $9(320 \mathrm{mg}, 66 \%, 0.89 \mathrm{mmol})$ as a yellow amorphous solid. - M. p. $85{ }^{\circ}$ C. $-R_{f}=0.54$ (ethyl acetate). $-{ }^{1} \mathrm{H}$ NMR $\left(500 \mathrm{MHz}, \mathrm{CDCl}_{3}\right): \delta=7.49-7.46(\mathrm{~m}, 2 \mathrm{H} ; \mathrm{H}$-aromat), $7.42-7.39$ (m, 1H; H-aromat), 7.31 (d, $2 \mathrm{H},{ }^{3} J=7.5 \mathrm{~Hz}$; $\mathrm{H}$-aromat), $4.95\left(\mathrm{~d}, 1 \mathrm{H},{ }^{3} J=7.5 \mathrm{~Hz} ; 8-\mathrm{H}\right), 4.35$ (br d, $1 \mathrm{H} ; 3-\mathrm{H}), 4.26\left(\mathrm{~d}, 1 \mathrm{H},{ }^{3} J=7.5 \mathrm{~Hz} ; 7-\mathrm{H}\right), 4.19(\mathrm{~d}, 1 \mathrm{H}$, $\left.{ }^{2} J=17.3 \mathrm{~Hz} ; \mathrm{NCH}_{2} \mathrm{COOCH}_{3}\right), 4.13\left(\mathrm{~d}, 1 \mathrm{H},{ }^{2} J=17.3 \mathrm{~Hz}\right.$; $\mathrm{NCH}_{2} \mathrm{COOCH}_{3}$ ), $4.10-4.04$ (ddd, $1 \mathrm{H},{ }^{2} \mathrm{~J}=11.7 \mathrm{~Hz},{ }^{3} \mathrm{~J}=$ $\left.4.0 \mathrm{~Hz},{ }^{3} \mathrm{~J}=4.9 \mathrm{~Hz} ; 5-\mathrm{H}\right), 3.75\left(\mathrm{~s}, 3 \mathrm{H} ; \mathrm{COOCH}_{3}\right), 3.67-$ $3.64\left(\mathrm{dd}, 1 \mathrm{H},{ }^{2} J=11.3 \mathrm{~Hz},{ }^{3} J=4.0 \mathrm{~Hz} ; 6-\mathrm{H}\right), 3.51-$ $3.45\left(\mathrm{ddd}, 1 \mathrm{H},{ }^{2} J=11.3 \mathrm{~Hz},{ }^{3} J=4.9 \mathrm{~Hz},{ }^{3} J=4.7 \mathrm{~Hz}\right.$; $\left.6^{\prime}-\mathrm{H}\right), 3.18-3.15\left(\mathrm{dd}, 1 \mathrm{H},{ }^{2} J=11.7 \mathrm{~Hz},{ }^{3} J=4.7 \mathrm{~Hz}\right.$; $\left.5^{\prime}-\mathrm{H}\right) .-{ }^{13} \mathrm{C} \mathrm{NMR}\left(125 \mathrm{MHz}, \mathrm{CDCl}_{3}\right): \delta=174.0(\mathrm{C}=\mathrm{O})$, $173.6(\mathrm{C}=\mathrm{O}), 168.8\left(\mathrm{C}=\mathrm{O}, \mathrm{NCH}_{2} \mathrm{COOCH}_{3}\right), 166.7(\mathrm{C}=\mathrm{O}$, 2-oxo-piperazine), 131.2 (q; C-aromat), 129.3 (ortho-C), 129.1 (para-C), 126.4 (meta-C), 75.2 (C-8), 66.3 (C-3), 52.6 $\left(\mathrm{COOCH}_{3}\right), 52.1(\mathrm{C}-7), 48.7\left(\mathrm{NCH}_{2} \mathrm{COOCH}_{3}\right), 46.1$ (C-6), 43.2 (C-5). For the signal assignments ${ }^{1} \mathrm{H},{ }^{1} \mathrm{H}-\mathrm{COSY}$ and HMQC spectra were recorded. - IR (KBr): $v=2953,1721$ (br, $\mathrm{C}=\mathrm{O}$, ester, imide), 1656 (C=O, 2-oxo-piperazine), 1597, 1495, 1438, 1389, 1350, 1250, 1206, 1069, 1024, 939, 869, 769, 733, 710, 693, $602 \mathrm{~cm}^{-1}$. - MS (EI, $\left.180{ }^{\circ} \mathrm{C}\right): \mathrm{m} / \mathrm{z}$ $(\%)=360(20)\left[\mathrm{M}^{+}+1\right], 359(100)\left[\mathrm{M}^{+}\right], 300(16), 272(12)$, 211 (50), 184 (17), 173 (29), 158 (44), 125 (32), 119 (26), 56 (38). - HR-MS (EI): calcd. 359.1116; found 359.1118. $\mathrm{C}_{17} \mathrm{H}_{17} \mathrm{~N}_{3} \mathrm{O}_{6}$ (359.34): calcd. C 56.82, H 4.77, N 11.60; found C 57.01, H 4.61, N 11.43.

General procedure for automated solid phase synthesis according to Scheme 4: Resin 11 (200 mg, $0.16 \mathrm{mmol}$, $0.78 \mathrm{mmol} / \mathrm{g}$ ) was swollen in dioxane ( $3 \mathrm{ml}, 1 \mathrm{~min}, 1 \times)$, the solvent removed in vacuo and the resin dried $(0.5 \mathrm{~min})$. After the addition of dioxane $(1.0 \mathrm{ml})$, a $0.85 \mathrm{M}$ solution of the $N$-substituted maleic imide in dioxane $(2.75 \mathrm{ml}$, $2.34 \mathrm{mmol}, 15.0$ equiv.) was added. The reaction mixture has heated to $65{ }^{\circ} \mathrm{C}$ for $2 \mathrm{~h}$. Then the solvent was removed in vacuo and dried in vacuo $(0.5 \mathrm{~min})$, washed twice with THF ( $3 \mathrm{ml}, 1 \mathrm{~min}, 1 \times)$ and dried $(0.33 \mathrm{~min})$. For reductive $\mathrm{N}-\mathrm{O}$ bond cleavage the resins were swollen in acetonitrile ( $3 \mathrm{ml}, 1 \mathrm{~min}, 1 \times$ ). Then the solvent was removed in vacuo and the resin was dried $(0.5 \mathrm{~min})$. After the addition of acetonitrile $(3.30 \mathrm{ml})$, water $(0.20 \mathrm{ml})$ and $\mathrm{Mo}(\mathrm{CO})_{6}(164 \mathrm{mg}$, $0.62 \mathrm{mmol}, 4.0$ equiv.) the reaction mixture was heated to $85^{\circ} \mathrm{C}$ for $3 \mathrm{~h}$. Afterwards the solvent was removed in vacuo, and the resin was dried $(1.0 \mathrm{~min})$ prior to three times washing with acetonitrile ( $3 \mathrm{ml}, 1 \mathrm{~min}, 2 \times)$ and drying ( $0.5 \mathrm{~min})$, followed by washing with $\operatorname{THF}(3 \mathrm{ml}, 1 \mathrm{~min}, 2 \times)$ and drying $(0.5 \mathrm{~min})$ and washing with methanol $(3 \mathrm{ml}, 1 \mathrm{~min}, 2 \times)$ and drying $(0.5 \mathrm{~min})$.

For the cleavage of the 1,3-aminoalcohols the resins (50 mg, $0.03-0.04 \mathrm{mmol}, 0.65-0.72 \mathrm{mmol} / \mathrm{g}$ ) were swollen in $4 \mathrm{ml}$ of THF and $0.5 \mathrm{ml}$ of methanol $(5 \mathrm{~min})$. After the addition of neat $\mathrm{NaOMe}(6.3 \mathrm{mg}, 0.12 \mathrm{mmol}, 3.33-3.64$ equiv.) the reaction mixture was stirred at room temperature for $22 \mathrm{~h}$. Afterwards the solvent was removed in vacuo and the resin was washed with methanol $(1.0 \mathrm{ml}, 4 \mathrm{~min}, 1 \times)$. The combined filtrates were dried in a speed vac concentrator. The crude products were analyzed by analytical RPHPLC/MS eluting with acetonitrile/water $5: 95+0.1 \%$ TFA $\rightarrow$ acetonitrile/water $100: 0+0.1 \%$ TFA within $5 \mathrm{~min}$.

Spectroscopic data of compound 12a: yellow oil. $-R_{f}=$ 0.13 (ethyl acetate). $-{ }^{1} \mathrm{H} \mathrm{NMR}\left(200 \mathrm{MHz}, \mathrm{CDCl}_{3}\right): \delta=7.57$ (d, $2 \mathrm{H},{ }^{4} \mathrm{~J}=1.96 \mathrm{~Hz}$; H-aromat), 7.09 (m, 1H; H-aromat), $4.98\left(\mathrm{~d}, 1 \mathrm{H},{ }^{3} \mathrm{~J}=8.78 \mathrm{~Hz} ; \mathrm{CH}\right), 4.30\left(\mathrm{~d}, 1 \mathrm{H},{ }^{3} \mathrm{~J}=8.78 \mathrm{~Hz}\right.$; 
$\mathrm{CH}), 4.22$ (br m, 3H; $\mathrm{CH}, \mathrm{CH}_{2}$ ), $3.81\left(\mathrm{~s}, 3 \mathrm{H} ; \mathrm{CH}_{3}\right), 3.77-$ $3.61\left(\mathrm{~m}, 3 \mathrm{H} ; \mathrm{CH}_{2}\right), 3.47-3.24\left(\mathrm{~m}, 1 \mathrm{H}, \mathrm{CH}_{2}\right)$. The signals of the secondary amine and the hydroxy group were not detectable. - IR (ATR): $v=3455(\mathrm{OH}), 3315(\mathrm{NH})$, 3185, 3082, 2954, 1747 ( $\mathrm{C}=\mathrm{O}$, ester), 1690 (br, $2 \mathrm{C}=\mathrm{O}$, imide), 1652 ( $\mathrm{C}=\mathrm{O}, 2$-oxo-piperazine), 1588, 1544, 1487, $1443,1412,1366,1306,1209,1182,1136,1114,994,985$, $927,844,801,763,723,670 \mathrm{~cm}^{-1}$. - MS (EI, $\left.230^{\circ} \mathrm{C}\right) \mathrm{m} / \mathrm{z}$ $(\%)=433(10)\left[\mathrm{M}^{+}+4\right], 431(64)\left[\mathrm{M}^{+}+2\right], 430(20)$
$\left[\mathrm{M}^{+}+1\right], 429(96)\left[\mathrm{M}^{+}\right], 415(14), 413(38), 251(56)$, 240 (28), 223 (66), 211 (26), 180 (32), 163 (62), 161 (100), 125 (24), 83 (26), 71 (32), 57 (56). - HR-MS (EI): calcd. for $\mathrm{C}_{17} \mathrm{H}_{17} \mathrm{~N}_{3} \mathrm{O}_{6} \mathrm{Cl}_{2}$ : 429.0494; found 429.0491 .

\section{Acknowledgements}

This work was supported by the BASF AG, the Deutsche Forschungsgemeinschaft and the Fonds der Chemischen Industrie.
[1] F. Wierschem, K. Rück-Braun, Eur. J. Org. Chem. 2321 (2004) and references cited therein. For details, see the supporting information.

[2] S. Maignan, J.P. Guilloteau, Y.M. Choi-Sledeski, M. R. Becker, W. R. Ewing, H.W. Pauls, A. P. Spada, V. Mikol, J. Med. Chem. 46, 685 (2003); S. Kitamura, H. Fukushi, T. Miyawaki, M. Kawamura, Z. Terashita, H. Sugihara, T. Naka, Chem. Pharm. Bull. 9, 258 (2001).

[3] A. Benjahad, R. Benhaddou, R. Granet, M. Kaouadji, P. Krausz, S. Piekarski, F. Thomasson, C. Bosgiraud, S. Delebasssée, Tetrahedron Lett. 35, 9545 (1994).

[4] C. J. Dinsmore, J. H. Hutchinson, T. M. Williams PCT Int. Appl. WO 99 09, 985 (Cl. A61 K31/495) (1999), GB Appl. 98/975 (1998); B. Baudoin, N. Dereu, Y. ElAhmad, P. Jimonet, A. Le Brun PCT Int. Appl. WO 99 41, 242 (Cl. C07D241/08) (1999), U.S. Appl. PV 81, 602 (1998).

[5] T.P. Burkholder, M.E. Kudlacz, Le Tieu-Binh PTC Int. Appl. WO 96 28,441 (Cl. C07 D403/06) (1996);
U. S. Appl. 404, 788 (1995); M. Matsuo, D. Hagiwara, H. Miyake PTC Int. Appl. WO 96 37,488 (Cl. C07 D403/06) (1996); GB Appl.95/10, 600 (1995).

[6] K. Rück-Braun, T.H.E. Freysoldt, F. Wierschem, Chem. Soc. Rev. 34, 507 (2005).

[7] S. I. Murahashi, H. Mitsui, T. Shiota, T. Tsuda, S. Watanabe, J. Org. Chem. 95, 1736 (1990); F. A. Davis, S. Chattopadhyay, J.C. Towson, S. Lal, T. Reddy, J. Org. Chem. 53, 2087 (1988).

[8] O. Tamura, K. Gotanda, J. Yoshino, Y. Morita, R. Terashima, M. Kikushi, T. Miyawaki, N. Mita, M. Yamashita, H. Ishibashi, M. Sakamoto, J. Org. Chem. 65, 8544 (2000); J. Marcus, J. Brussee, A. V. D. Gen, Eur. J. Org. Chem. 2513 (1998).

[9] S. Cicchi, A. Goti, A. Brandi, A. Guarna, F. De Sarlo, Tetrahedron Lett. 31, 3351 (1990); B. M. Trost, L. S. Chupak, T. Lübbers, J. Am. Chem. Soc. 120, 1732 (1998); J. W. Bode, E. M. Carreira, J. Org. Chem. 66, 6410 (2001). 